EPJ Web of Conferences 16, 03006 (2011)

DOI: $10.1051 /$ epjconf/20111603006

(C) Owned by the authors, published by EDP Sciences, 2011

\title{
Post-processing of high-contrast observations of exoplanets
}

\author{
S. Gladysz ${ }^{a}$
}

\author{
European Southern Observatory, Karl-Schwarzschild-Strasse 2, 85748 Garching, Germany
}

\begin{abstract}
Post-processing of images delivered by the eXtreme Adaptive Optics (XAO) instrumentation is a crucial step which can increase achievable contrast even by two orders of magnitude. In this communication I present a new class of algorithms for detection of extrasolar planets from a sequence of adaptive-opticscorrected images. In general, the methods discriminate between real sources and stellar PSF features based on statistics of recorded intensity. The methods are particularly useful in dealing with static speckles which are the greatest obstacle in detecting exoplanets.
\end{abstract}

\section{INTRODUCTION}

The main limitation in astronomical imaging of faint, sub-stellar companions to stars is the contrast ratio and small separation between the two components of the system, so that the companion is hidden in the glare of the star. This is the reason why most discoveries of exoplanets have been made indirectly. Future XAO systems coupled with efficient coronagraphy will mitigate the effects of turbulent atmosphere and diffraction by the telescope's aperture; nevertheless clever post-processing is required in order to detect the faintest planets.

Recently a new class of reference-less, multi-frame methods has been proposed for this problem [1]. The methods rely on statistics of intensity rather than its spatial distribution in the image plane. The latter can be often misleading in high-contrast AO imagery, because static aberrations in the optical system produce static speckles in the final image which look exactly like real sources. The new methods rely on the observation that in AO imagery light from a planet varies less than a quasi-static speckle. This relative difference in intensity variability can be estimated from multi-frame data, and quantified using optimal discriminators.

\section{RESULTS}

With multi-frame data one can compute parameters of intensity distributions in a data cube. The parameters are chosen so that stellar light is suppressed while light from a companion is amplified. As an example we map local ratios of the parameters of the Rician distribution, which has been traditionally used to model AO speckle.

Figure 1 shows the results of post-processing of AO observations from three telescopes: $5 \mathrm{~m}$ Hale Telescope (with the LAMP camera, 25\% Strehl ratio), $3 \mathrm{~m}$ Shane Telescope (with the IRCAL detector, $50 \%$ Strehl ratio), and the $8 \mathrm{~m}$ VLT (with VISIR, $80 \%$ Strehl ratio). Figure 2 shows how the new method

\footnotetext{
ae-mail: sgladysz@eso.org
}

This is an Open Access article distributed under the terms of the Creative Commons Attribution-Noncommercial License 3.0, which permits unrestricted use, distribution, and reproduction in any noncommercial medium, provided the original work is properly cited. 


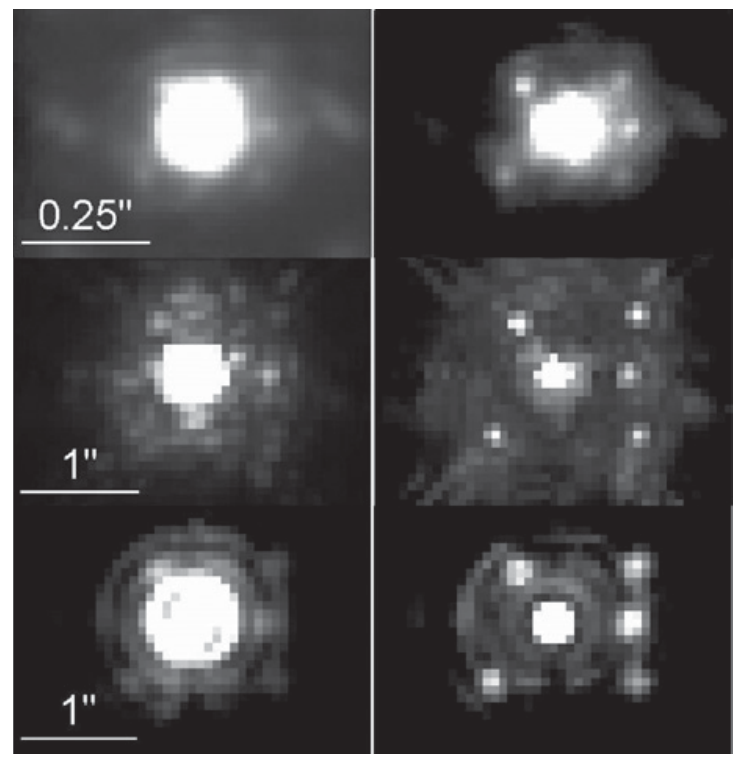

Figure 1. Top to bottom: Palomar, Lick and VLT observations. In each case five artificial companions were inserted in the long exposures (shift-and-add images) as well as in the data cubes, in order to compare classical and "statistical" imaging. Left column: long exposures. Right column: statistical maps. In many cases sources which were hidden in the shift-and-add images are now very clearly seen. Images are displayed on the linear scale; the central parts are deliberately saturated to bring out the faint speckle structure outside the PSF core.
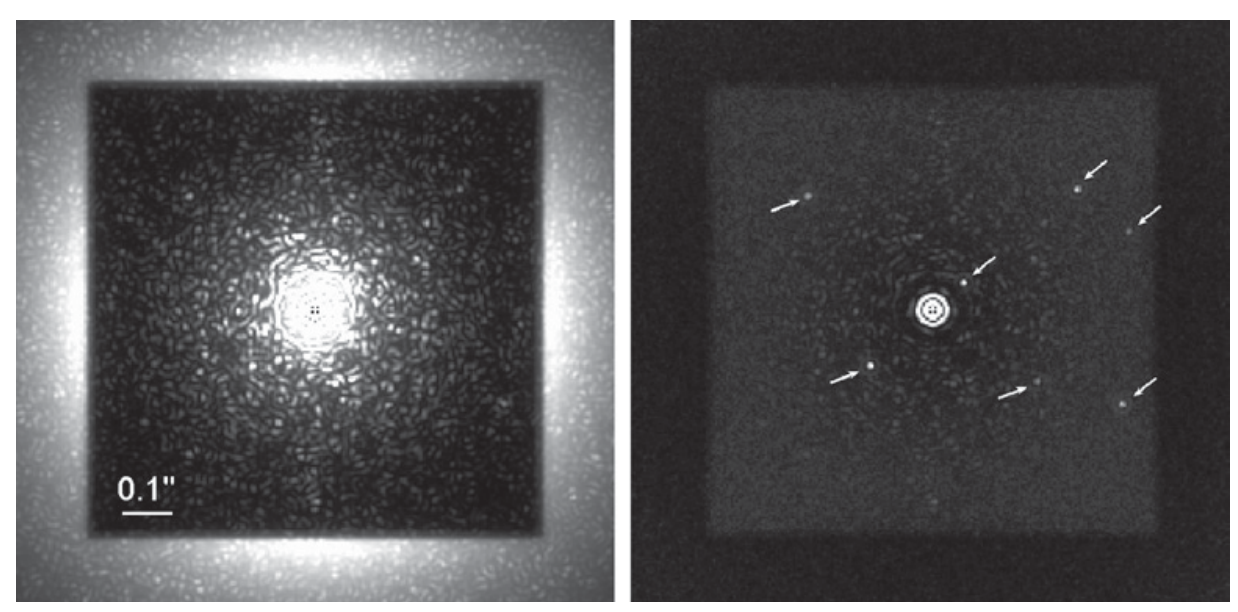

Figure 2. Simulated observations with the E-ELT: coronagraphic data cube of 100 images, each 1sec-long, with seven artificial planets (contrast ratios between $5 \cdot 10^{-5}$ to $2 \cdot 10^{-6}$ ). Left: long exposure (shift-and-add). Right: statistical map for the same data.

compares to classical, long-exposure imaging for simulated observations with the $42 \mathrm{~m}$ European Extremely Large Telescope (E-ELT).

\section{References}

[1] Gladysz, S., Christou, J., Astrophysical Journal 698, (2009) 28 